\title{
Septic arthritis of the hip with intraperitoneal dislocation of the femoral head
}

\author{
Eloka O. Okoye', Abdussemee I. Aburrazzaaq ${ }^{1}$, Abraham A. Anejukwo², Taiwo A. Lawal' \\ 1. Department of Surgery, University College Hospital Ibadan, Nigeria \\ 2. Department of Orthopaedics and Trauma Surgery, University College Hospital, Ibadan, Nigeria \\ Correspondence: DrTaiwo A. Lawal (taiwo.lawal@hotmail.com)
}

(c) 2018 E.0. Okoye et al. This open access article is licensed under a (reative Commons Attribution 4.0 International License (http://creativecommons.org/licenses/by/4.0/) which permits unrestricted use, distribution, and reproduction in any medium, provided you give appropriate credit to the original author(s) and the source, provide a link to the Creative Commons license, and indicate if changes were made.

East Cent Afr J Surg. 2018 Dec;23(3):123-125 https://dx.doi.org/10.4314/ecajs.v23i3.7

\begin{abstract}
Septic arthritis is a potentially crippling infection of the joint cavity. Malnutrition in the setting of poor socioeconomic background is a known predisposing and propagating factor of septic arthritis. Prolonged untreated septic arthritis of the hip joint can be a rare cause of acute abdomen, this is due to the proximity of the hip joint to the pelvis causing the spread of the causative organisms into the peritoneal cavity.
\end{abstract}

We present a case of septic arthritis of the hip joint presenting as acute abdomen with intra-peritoneal dislocation of the head of the femur, and reviewed relevant literature.

Keywords: acute abdomen, septic arthritis, peritonitis, fracture dislocation

\section{Introduction}

Acute abdomen is an abdominal condition of sudden onset that may require immediate operative intervention. ${ }^{1}$ Surgical treatment often is required after an initial period of active resuscitation since many of the patients present late as a result of pre-hospital delay. ${ }^{2}$

Acute appendicitis, intestinal obstruction and perforated typhoid ileitis are the commonest causes of acute abdomen in sub-Saharan Africa. ${ }^{3}$ Septic arthritis of the hip, on very rare occasions can cause acute abdomen and only two cases have been documented in the literature. ${ }^{4}$

We hereby present a case of septic arthritis presenting as acute abdomen, with intra-peritoneal dislocation of the head of the femur and reviewed relevant literature.

\section{Case presentation}

A 7-year-old female from a low-income family was seen at the children emergency department with a left gluteal swelling, fever, generalized abdominal pain and progressive abdominal distension of three weeks duration.

On examination, she was acutely ill looking, pale, and had tachycardia and tachypnoiea. She had a grossly distended abdomen with an infra-umbilical sinus copiously discharging pus (Figure 1), and bilateral lower limb oedema, with the left much more swollen than the right limb. A clinical diagnosis of perforated typhoid ileitis complicated with empyema abdominis necessitans was made.
She was actively resuscitated and optimized for exploratory laparotomy. The findings at surgery were pus in the subcutaneous tissues tracking towards the gluteal region, multiple intra-peritoneal fibrous adhesions, organized pus in the pelvis, and a round bony structure (with a surrounding pyogenic membrane) freely floating in the left paracolic gutter (Figure 2). There was no intestinal perforation. She had peritoneal lavage with warm normal saline and mass closure done for the abdomen.

Postoperatively, radiographs done revealed a left hip dislocation with absent left femoral head. The left lower limb was immobilized with skin traction. She made a slow but progressive recovery, and four weeks after surgery, the skin traction was discontinued. She was discharged to the outpatient clinic, but was lost to follow up after an initial two weeks period.

\section{Discussion}

Septic arthritis is an acute infection of the joint space, and in children presents with high grade continuous fever, dehydration, tachycardia, joint swelling and tenderness. ${ }^{3}$ Septic arthritis is typically caused by hematogenous spread from an infection of the skin or mucous membrane, but can also arise from direct extension from trauma to the joint or bone. ${ }^{5}$ The most commonly infected joints are the knee (41\%), hip (20)\%, ankle (14\%) and elbow (12\%) joints. ${ }^{5}$ Factors such as systemic viral illness (chicken pox, respiratory 


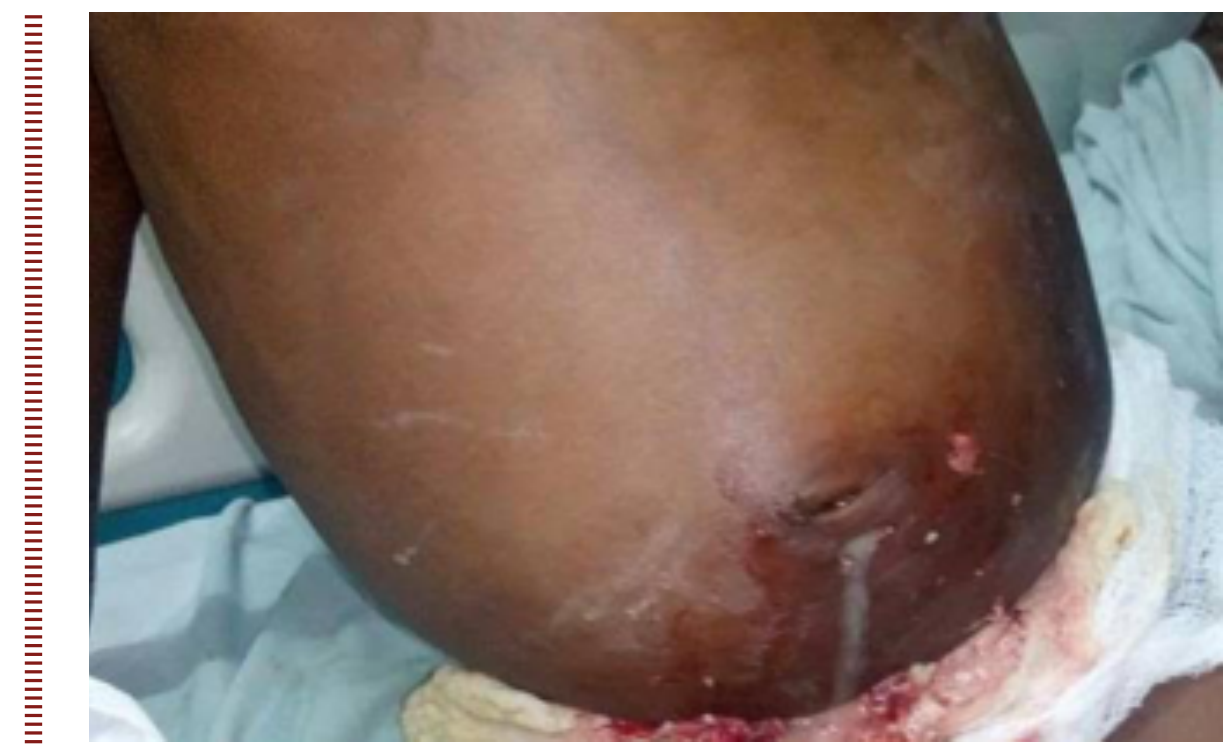

Figure 1. Grossly distended abdomen with an infra-umbilical sinus discharging pus

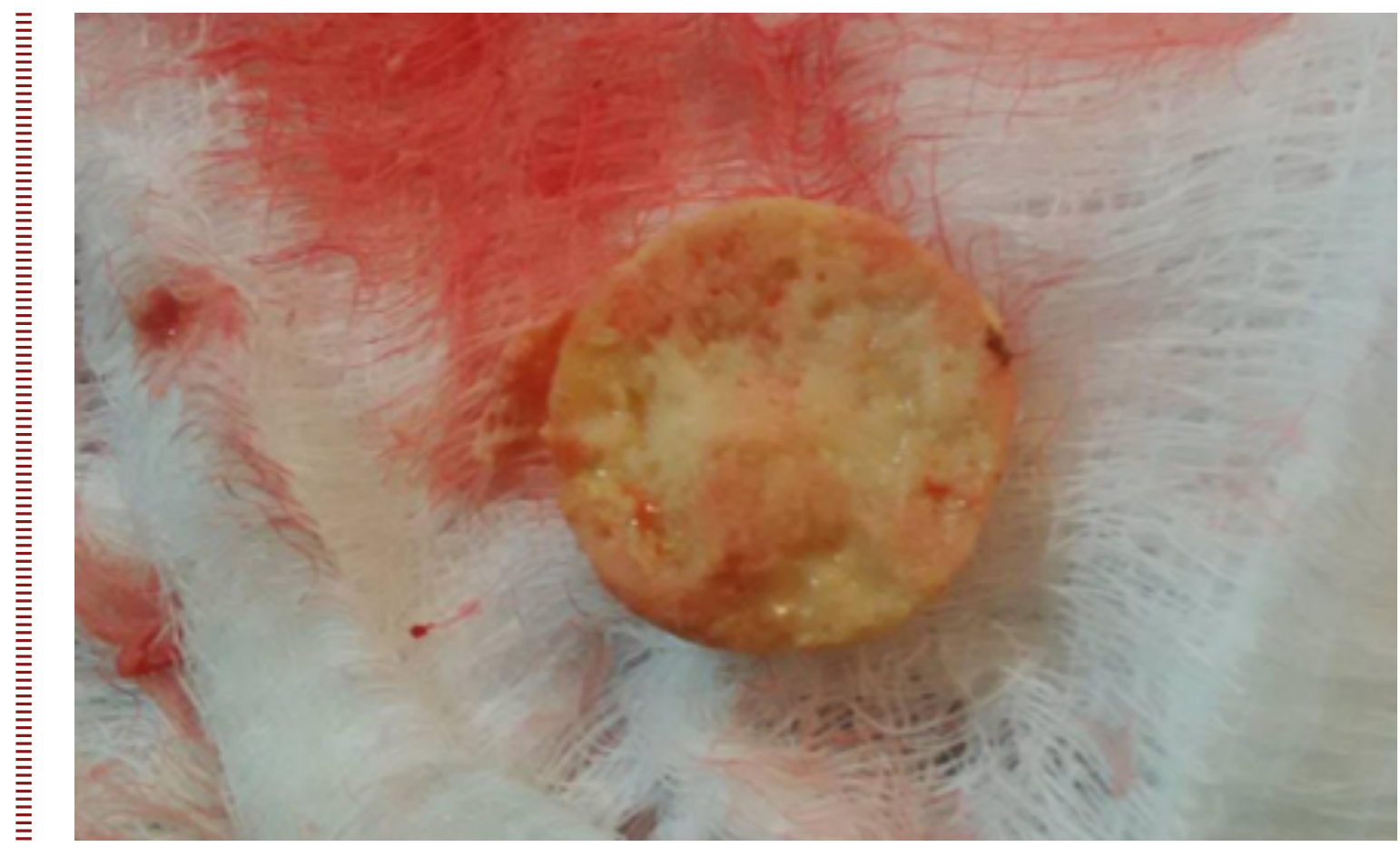

Figure 2. Dislocated femoral head found in the peritoneal cavity

or urinary tract infection), immunosuppressive disorders or sickle cell disease increase the susceptibility to septic arthritis. ${ }^{5}$ Although a risk factor was not identified in this case, it could have followed an indolent viral illness. Malnutrition consequent on the patient's socioeconomic background could have been contributory.

The presence and multiplication of the offending organism in the joint cavity evokes an acute inflammatory response which results in migration of polymorphonuclear cells, production of cytokines and proteolytic enzymes, with attending degradation of articular cartilage and suppuration. ${ }^{5,6}$ As the infection progresses and more of the joint is destroyed, the accumulated pus may form a sinus which discharges through the skin.

The diagnosis of septic arthritis should be made promptly to prevent damage to the articular cartilage. Blood and synovial fluid should be taken for microscopy culture and sensitivity although the identification of purulent joint effusion is diagnostic. Plain radiographs of the joints may show widening of the joint space, effusion or dislocation. The 
mainstay of treatment is joint decompression or debridement, which may be through percutaneous or open surgical means. Intravenous antibiotics and early joint mobilization are imperative adjuncts. ${ }^{4,5,7}$

The hip joint is a multiaxial ball and socket synovial joint between the head of the femur and the acetabulum. The acetabular fossa is related medially to the pelvis and is only separated from it by obturator internus and peritoneum. This proximity of the hip joint to the pelvis favours internal drainage of the pus into the peritoneal cavity as seen in the index patient. ${ }^{8}$

If untreated, septic arthritis is crippling in children and in the hip causes rapid destruction the femoral head due to a disruption of its blood supply. ${ }^{7}$

This case showed that the pus in the joint cavity can track into the pelvis causing generalized peritonitis and may even extrude the upper femoral epiphysis into peritoneal cavity. The acetabular fossa is closely related to the pelvic cavity and is only seperated from it, by the obturator internus muscle, its fascia and the peritoneum. Pus accumulating within the acetabular fossa can track through the obturator internus muscle and rupture into the pelvis. Infected fluid within the peritoneal cavity can dissect through the soft tissues of the anterior abdominal wall creating a subcutaneous abscess. This abscess ruptures through the skin creating a discharging sinus as seen in the index case. We hope that this report will add to literature, and will encourage that index of suspicion in children who present with similar features.

\section{Competing interests}

All authors declare that they have no competing interests related to this work.

\section{References}

1. EA Badoe, EQ Achampong J da R-A. The acute abdomen. In: Principles and practice of surgery including pathology in the tropics. Fourth. p. 534-51.

2. Faquharson M MB. Emergency laparotomy. In: Faquharsons textbook of operative surgery. Fourth. 2005. p. 233-46.

3. EA Badoe, EQ Achampong J da R-A. Principles and practice of surgery including pathology in the tropics. 546-551 p.

4. TO Alonge, SO Ogunlade, AB Omololu MO. Septic Arthritis of the Hip Joint presenitng as Acute Abdomen- a Report of Two Cases. Niger J Paediatr. 2003;30.

5. Emmanuel A. Ameh, Stephen W. Bickler KL, Benedict C. Nwomeh DP. Haematogenous osteomyelitis and septic arthritis. In: Paediatric Surgery: A Comprehensive Text for Africa. 2011. p. 135-41.

6. Kumar V, Abbas A AJ. Infectious arthritis. In: Robbins \& Cotran Pathologic Basis of Dlsease. Ninth. 2015. p. 1213-5.

7. EA Badoe, EQ Achampong J da R-A. Orthopaedic surgery. In: Principles and practice of surgery including pathology in the tropics. Fourth. p. 1132-90.

8. Sinnatamby CS. Gluteal region and hip joint. In: Last's anatomy; Regional and applied. Twelvth. 2011. p. 122-9. 